\title{
Estrategia de Bioética en el Plan Integral de Salud Mental de Andalucía.
}

Bioethical strategy in the comprehensive mental health plan of Andalucia.

\author{
Rafael del Pino López a \\ ${ }^{a}$ Médico Psiquiátra. Director Unidad de Gestión Clínica de Salud Mental. Hospital Universitario Virgen \\ de la Victoria. Málaga, España.
}

Correspondencia: Rafael del Pino López.(rafael.pino.lopez@gmail.com)

Recibido: 12/05/2014; aceptado: 31/12/2014

RESUMEN: Las personas que padecen un trastorno mental pueden sufrir problemas de exclusión social y sus derechos humanos pueden ser violados en diferentes esferas. Por paternalismo, prejuicio o ignorancia, esta situación puede darse también en la atención que prestan los profesionales.

La provisión de servicios profesionales de calidad implica un enfoque que promueva el conocimiento y la protección de los derechos fundamentales de las personas atendidas, facilitándose así la lucha contra el estigma y el desarrollo de un modelo de recuperación. A través del presente artículo de revisión:

1. Se Reflexiona sobre la protección de los derechos y las consecuencias sobre el estigma y la recuperación de las personas que padecen un trastorno mental.

2. Son considerados algunos de los aspectos relacionados con la capacidad, el consentimiento informado y la planificación anticipada de decisiones.

3. Se plantean elementos de discusión sobre el Tratamiento Ambulatorio Involuntario (TAI) y sus posibles alternativas.

PALABRAS CLAVE: Bioética. Trastornos Mentales. Derechos Humanos. Estigma Social. Consentimiento Informado. Autonomía Personal.
ABSTRACT: Persons with a mental disorder may suffer problems of social exclusion and their rights can be violated in different spheres. By paternalism, ignorance or prejudice, this situation can also occur in the care provided by professionals. The provision of high quality professional services implies an approach that promotes the knowledge and the protection of the fundamental rights of persons attended, facilitating in this way the fight against stigma and the development of a recovery model. The aim in though this review is:

1. Reflect on the protection of the rights and the consequences of stigma in the recovery process of persons suffering from a mental disorder.

2. Consider some aspects related to capacity, informed consent and the Psichiatric Advance Directives.

3. Bring up elements for discussion on involuntary outpatient treatment and possible alternatives.

KEY WORDS: Bioethics. Mental Disorders. Human Rights. Social Stigma. Informed Consent. Personal Autonomy.

Agradecimientos:

A la Escuela Andaluza de Salud Pública y al Grupo Intersectorial de Derechos Humanos y Salud Mental $^{2}$, dependiente del II Plan Integral de Salud Mental de Andalucía, que vienen trabajando comprometida e ininterrumpidamente en tareas de revisión, formación y asesoramiento sobre los derechos de las personas que padecen trastornos mentales y son atendidas en el Sistema Sanitario Público Andaluz.

\footnotetext{
2 Grupo de Derechos Humanos y Salud Mental. II PISMA. José Manuel Arévalo López (Usuario. Presidente Federación Andaluza de Asociaciones de Usuarios/as de Salud Mental En Primera Persona), María José Ariza Conejero (Enfermera especialista en salud mental. Unidad de Gestión Clínica de Salud Mental Hospital de Virgen de Valme. Servicio Andaluz de Salud), Águila Bono del Trigo (Socióloga. Profesora Área Ciudadanía, Ética y Participación. Escuela Andaluza de Salud Pública), Carlos Dueñas Abril (Psiquiatra. Unidad de Gestión Clínica de Salud Mental. Hospital de Virgen Macarena. Servicio Andaluz de Salud), Pablo García-Cubillana de la Cruz (Enfermero especialista en salud mental y Psicólogo. Programa de Salud Mental. Servicio Andaluz de Salud), Evelyn Huizing (Enfermera especialista en salud mental. Programa de Salud Mental. Servicio Andaluz de Salud), Vicente Ibáñez Rojo (Psiquiatra. Unidad de Gestión Clínica de Salud Mental Hospital Torrecárdenas. Servicio Andaluz de Salud), Ana Martín Mingorance (Abogada. FEAFESAndalucía), Bibiana Navarro Matilla (Psicóloga. Profesora colaboradora de la Escuela Andaluza de Salud Pública), Javier Romero Cuesta (Psiquiatra. Unidad de Gestión Clínica de Salud Mental Intercentros de la provincia de Huelva), Fernando Santos Urbaneja (Fiscal. Fiscalía de Córdoba), Teresa del Sol Chicharro (Terapeuta Ocupacional. Ex-Responsable Provincial de Málaga. Fundación Pública Andaluza para la Integración Social de Personas con Enfermedad Mental).
} 
"Si nos hundimos antes de nadar no soñarán los peces con anzuelos,

Si nos rendimos para no llorar, declarará el amor huelga de celo."

JOAQUÍN SABINA

\section{Introducción}

Históricamente, la institucionalización manicomial, al margen de pretendidas funciones terapéuticas, supuso una barrera de protección social respecto al "loco", exento este de los derechos más fundamentales. La estrecha asociación histórica entre movimientos de cambio social y político y los distintos procesos de reforma psiquiátrica, encuadran un contexto de búsqueda continua de alternativas a la "institución total", a través de complejos sistemas de atención en la comunidad, desarrollándose un progresivo consenso técnico en relación, tanto a tipos de intervenciones efectivas, como a estructuras y organización de los recursos sanitarios y sociales que deben sustentarlas.

Aunque existen diferencias conceptuales y organizativas en los distintos sistemas de atención a los problemas de salud mental, desde un Modelo de Atención Comunitario destacan el enfoque de compromiso en la recuperación, una red de dispositivos, (que incluye tanto servicios sanitarios, como sociales), y el trabajo de equipos multidisciplinares, que aseguran un abordaje integral del trastorno mental y garantizan el respeto a los derechos fundamentales de las personas atendidas.

Pero, la mera existencia de códigos deontológicos, leyes y normas no aseguran que se lleve a cabo una práctica asistencial ética por parte de los profesionales sanitarios y sociales, incluidos los de salud mental. Además, la mayor parte de las violaciones de los derechos de las personas que padecen un trastorno mental, a través de prácticas erróneas o aplicación inadecuada de protocolos, se llevan a cabo sin intención ni conciencia de que se está violando un derecho. Velar por el respeto a los derechos de las personas atendidas en los servicios sanitarios y sociales no significa culpabilizar a los profesionales, sino hacer conscientes a todos los implicados de que se puede estar incumpliendo la normativa vigente (1).

Para avanzar en una cultura ética a nivel de los servicios asistenciales y favorecer una conciencia de responsabilidad en el comportamiento ético de los profesionales se consideran claves la formación y el debate permanentes, al igual que el adecuado desarrollo normativo que suponga mayores garantías para la aplicación práctica de todos los derechos (apoyos para la toma de decisiones que respeten la autonomía y las preferencias de las personas, consentimiento informado, ingresos involuntarios, uso de medidas coercitivas... etc.). Así mismo, importa la difusión entre las personas con problemas de salud mental de información sobre los dere- 
ORIGINALES Y REVISIONES

chos que legalmente les asisten y a qué instancias recurrir en caso de que estos sean vulnerados.

En el marco del II Plan Integral de Salud Mental de Andalucía (PISMA) (2), y a la vez de la primera edición de la Estrategia de Bioética del Sistema Sanitario Público Andaluz (SSPA) (3), se crea el Grupo Intersectorial de Derechos Humanos y Salud Mental, con el objetivo de analizar y proponer medidas de mejora que garanticen que los derechos humanos de las personas que padecen problemas de salud mental atendidas en el SSPA, sean respetados y protegidos, tal como se exige en la Convención sobre los Derechos de las Personas con Discapacidad (CDPD) (4). El presente artículo de revisión está condicionado en buena medida por el trabajo realizado por dicho Grupo y plasmado en el informe: "Derechos Humanos y Salud Mental en Andalucía" (5), que adelanta una aproximación estratégica sobre los derechos de las personas que padecen una enfermedad mental para ser desarrollada en un próximo PISMA.

\section{Justificación y objetivos}

En el II PISMA (2) se enfatiza la necesidad de proteger los derechos de las personas que padecen una enfermedad mental. Concretamente, en el quinto objetivo se expresa la necesidad de mejorar la calidad de vida de estas personas y de su entorno, velando por el cumplimiento de sus derechos.

Además, el II Plan de Acción Integral para Personas con Discapacidad de Andalucía (PAIPDA) (6), fue concebido de conformidad con la CDPD y algunos de sus principios se relacionan de forma especial con la protección de derechos: el respeto de la dignidad, la autonomía individual, la autodeterminación y la independencia de las personas, la no discriminación por motivos de discapacidad e igualdad de oportunidades, la calidad de vida (bienestar emocional, relaciones interpersonales, bienestar material, desarrollo personal, bienestar físico, autodeterminación, inclusión social y derechos), el respeto por la diferencia y la cooperación institucional.

En el Plan de Calidad 2006-2010 de la Fundación Pública Andaluza para la Integración Social de las Personas con Enfermedad Mental (FAISEM) (7), se establecieron, entre otros, como valores fundamentales: la atención al usuario basada en las necesidades globales y orientada a la igualdad y el respeto de los derechos de ciudadanía, en base a principios que incluyen el respeto de la intimidad, privacidad, confidencialidad, individualidad, autonomía y normalización.

Las posibles violaciones de los derechos de personas que padecen un Trastorno Mental Grave (TMG) no son exclusivas de instituciones cerradas, sino que pue- 
den ocurrir en el entorno de servicios sanitarios basados en la atención comunitaria, y también en el ámbito de servicios sociales generales o de apoyo específicos. La reproducción de prácticas manicomiales puede conllevar situaciones de maltrato, distrato, violencia y sufrimiento, incluso en el más estricto ámbito comunitario (8).

El mayor riesgo de atentar contra los derechos reside en la propia relación asimétrica que se estable entre los profesionales y las personas que necesitan asistencia y cuidados en salud mental (9). Sólo a través de una nueva cultura de práctica ética profesional, cuya base es el respeto hacia los derechos humanos de estas personas, podrán desterrarse abusos, que en muchas ocasiones pasan de manera desapercibida (1).

Como se recoge en el Libro Verde (10) de la Comisión de las Comunidades Europeas "Mejorar la Salud Mental de la Población. Hacia una estrategia de la Unión Europea en materia de Salud Mental": sin salud mental no hay salud. Para los ciudadanos constituye el recurso que les permite desarrollar su potencial intelectual y emocional, así como encontrar y desempeñar su papel en la sociedad, la escuela y el trabajo.

\section{Desarrollo metodológico}

\subsection{Estigma y Modelo de Recuperación}

\subsubsection{El estigma asociado a los trastornos mentales}

En el mundo occidental, una de cada cuatro personas padece una enfermedad mental a lo largo de su vida, lo que conlleva un elevado coste personal, social y económico. En muchos casos puede ser un proceso largo y doloroso, no sólo atendiendo a la gravedad de su patología, sino también por las consecuencias de la imagen negativa que la sociedad tiene de estas personas.

La percepción social de la enfermedad mental está sesgada por el desconocimiento y la desinformación, e influye en el aislamiento de quienes la padecen. El estigma de la enfermedad mental, sustentado en prejuicios y causante de discriminación social, se debe combatir por injusto, cruel y por no tener base científica (11). Este debate debe extenderse al ámbito de la propia sociedad, donde el escenario educativo toma una especial relevancia (12).

El estigma afecta directamente a la persona (disminuye su autoestima, favorece el aislamiento y dificulta la petición de ayuda), e indirectamente como barrera al acceso a servicios (empleo, vivienda, servicios sanitarios y sociales...) (13). También los profesionales sanitarios, incluidos los de salud mental, pueden ser 
ORIGINALES Y REVISIONES

fuente de estigmatización en la práctica asistencial diaria. Podría ser el caso de atribuir de forma errónea síntomas físicos al propio trastorno mental y no realizar las oportunas pruebas diagnósticas cuando, además, la evidencia demuestra que las personas que padecen una enfermedad mental tienen peor salud general que el resto de la población: mayor prevalencia, alta comorbilidad, mayores tasas de infecciones, de enfermedades cardiacas y respiratorias, mayor mortalidad (sobre todo cardiovascular) y peor acceso a servicios y cuidados.

Un ejemplo de intervención para luchar contra el trato discriminatorio de las personas que padecen una enfermedad mental, atendidas en distintos niveles del Sistema Sanitario, es el documental realizado en el seno de la Estrategia anti-estigma ldecada4 (14), donde se ponen de manifiesto las falsas creencias y prejuicios sobre la salud general de las personas que padecen una enfermedad mental.

En Andalucía, en relación a la lucha contra el estigma y a favor del enfoque de la recuperación de las personas que padecen un TMG, tienen actualmente un importante papel los Movimientos Asociativos de Familiares (15) y los Movimientos Asociativos de Usuarios (16).

\subsubsection{El modelo de recuperación}

La Organización Mundial de la Salud (OMS) advierte de que muchas violaciones de derechos de las personas que padecen una enfermedad mental pueden producirse en el ámbito de los propios servicios sanitarios, de modo que la atención prestada puede dificultar la recuperación de la persona a costa de que esté "bajo control", mermando su autonomía, la mejora de su bienestar y su calidad de vida (17).

Recuperación no significa curación en el sentido de desaparición de los síntomas o la vuelta a niveles de funcionamiento previos a la enfermedad, sino alcanzar una forma de vida en la que es posible sobreponerse a la propia enfermedad, asumiendo y definiendo una expectativa personal de vida.

En todo caso, el concepto de recuperación plantea el proyecto de vida, una vez han aparecido las consecuencias de la enfermedad. Supone apoyarse en las capacidades y fortalezas de la persona y de su entorno, para conseguir un proyecto rehabilitador que cuente con la participación del afectado y no esté exclusivamente definido por los estándares de salud que plantean los profesionales.

Una de las críticas más extendidas al enfoque de recuperación hace referencia a que aumentan los riesgos para los pacientes y las responsabilidades para los profesionales. Sin embargo, el riesgo es inherente a todo servicio de salud. En los servicios "orientados a la recuperación" los riesgos pueden aumentar, pero en ocasiones es necesario asumirlos. Deben distinguirse los riesgos que se deben minimizar, como por ejemplo de auto y heterolesiones, de los riesgos que las personas tienen derecho a experimentar. El enfoque de la recuperación fomenta las opor- 
tunidades de cambio y crecimiento, "la dignidad de arriesgarse", pero de forma responsable. (18).

El empoderamiento de las personas que padecen una enfermedad mental constituye un elemento esencial de cara a su recuperación, dirigido a ayudar en la autodeterminación, la autoestima y la autonomía personal (19). Empoderar debe significar promover autonomía, delegar poder de decisión y desarrollar capacidades. Este es el escenario donde la persona puede motivarse e implica una mejora de sus expectativas en el proceso terapéutico.

\subsection{Discapacidad, Salud Mental y Derechos Humanos}

Los tiempos cambian y con ellos han ido naciendo paulatinamente núcleos de opinión y pensamiento laicos que adoptan posturas y expresan opiniones sobre la visión de las personas con discapacidad, sobre sus derechos, su discriminación y sobre campos del pensamiento, entre los que se encuentra la bioética (20).

En 1975 la Asamblea General de la Organización de Naciones Unidas (ONU) propone la Declaración sobre los Derechos de las Personas Impedidas (21), desarrollándose posteriormente una serie de documentos para la protección y promoción de los derechos de las personas con discapacidad, como son en 1982 el Programa de Acción Mundial para las Personas Impedidas (22) y en 1993 los Estándares sobre la igualdad de oportunidades para las Personas con Discapacidad (23).

Sobre la protección de los derechos humanos de las personas que padecen una enfermedad mental destacan en la década de los 90 una serie de documentos específicos que, aunque sin garantizar el derecho al consentimiento de tratamiento y sin carácter vinculante a nivel legal, son referentes para desarrollos normativos y formulación de políticas para los distintos países (5): ONU: Principios para la Protección de las Personas con Enfermedades Mentales y la Mejora de la Atención de Salud Mental (24), OMS: Directrices sobre los derechos humanos de las personas con trastornos mentales (25), OMS: Atención a la salud mental: diez principios básicos (26).

A nivel europeo, y en relación a la protección de los derechos, es de destacar la Recomendación 2004 del Consejo de Europa (27), que consagra los principios de restricción mínima y establece criterios para la aplicación del internamiento y del tratamiento involuntario, exige el control médico de medidas coercitivas (aislamiento y contención) e introduce la importancia de la valoración ética.

La discapacidad, incluida la que sufren las personas con enfermedad mental, es un concepto que ha evolucionado con el tiempo. No sólo se entiende como un problema de la persona causado por la enfermedad, sino que son determinantes los obstáculos y las actitudes sociales del entorno, en relación a su participación plena y efectiva en la sociedad, incluyendo los deseos, metas y objetivos en su vida cotidiana (28). 
ORIGINALES Y REVISIONES

La Convención de la Organización de Naciones Unidas sobre los Derechos de las Personas con Discapacidad (CDPD), aprobada el 13 de diciembre de 2006 por la Asamblea General de las Naciones Unidas, fue ratificada por España y publicada en el Boletín Oficial del Estado el día 21 de Abril de 2008 (29).

La CDPD se aleja del modelo médico dominante y adopta un enfoque amplio del concepto de discapacidad, considerando las políticas relativas a las personas con discapacidad, no como una cuestión recomendable o altruista, sino como una absoluta exigencia basada en el respeto a todos los derechos humanos y las libertades, lo que supone el logro de muchas de las reivindicaciones históricas.

La Convención expone los principios de participación, respeto por la diferencia y la no discriminación. Refiere, así mismo, las obligaciones de los Estados sobre la necesidad de adoptar medidas legales en la lucha contra la discriminación, sobre la necesidad de formación de profesionales y personas cuidadoras, así como la importancia de sensibilizar a todos los implicados, incluidos familias y profesionales, sobre el respeto a los derechos y a la dignidad de las personas que padecen una discapacidad. Supone un nuevo enfoque sobre la discapacidad, haciendo especial hincapié en los derechos humanos de las personas y en los decisivos factores de exclusión que se plantean desde el ámbito social (30).

\section{Discusión}

\subsection{El Tratamiento Ambulatorio Involuntario}

Los Tratamientos Ambulatorios Involuntarios (TAI) en salud mental, con distintos desarrollos en las últimas décadas en el Reino Unido, Canadá y EEUU (31), se definen desde criterios diversos, pero precisan en todos los casos una clara situación clínica de incapacidad del paciente para acceder voluntariamente a un determinado tratamiento y su supervisión en el medio comunitario, de tal manera que el tratamiento forzoso pueda prevenir recaídas y evitar posibles daños al propio paciente $y / o$ a terceros. Cuando se plantean como una intervención preventiva en ausencia de crisis o aparente riesgo, continúan planteando un intenso debate.

La propuesta de la Federación de Asociaciones de Familiares (FEAFES) en 2006 de un proyecto de modificación de la Ley de Enjuiciamiento Civil (LEC), consistente en añadir un artículo más al 763: "podrá también el tribunal autorizar un tratamiento no voluntario por razón de trastorno psíquico o un periodo de observación para el diagnóstico, cuando así lo requiera la salud del enfermo, previa propuesta razonada del facultativo, audiencia del interesado, informe del forense y del Ministerio Fiscal", hace eclosionar en nuestro país un intenso debate entre 
partidos políticos, Asociación Española de Neuropsiquiatría (AEN) y Defensor del Pueblo. La posición de la AEN, apoyada en su día por la totalidad de los partidos políticos, fue clara (32): no asumir la promulgación de leyes específicas que puedan estigmatizar a personas que padecen trastornos mentales, sin suficiente evidencia científica, sin referencias en el marco Europeo sobre este tipo de reformas legales específicas y con serias dudas sobre las consecuencias de la "judicialización" del tratamiento a nivel de responsabilidad e implicación de profesionales y pacientes, tanto en el plan, como en el "contrato terapéutico".

Sorprende la escasa sensibilidad prestada a importantes aspectos éticos que se plantean en los TAI (33). La mayoría de las posiciones más proclives esconden una doble polémica: en relación a la peligrosidad de los pacientes y sobre la falta de recursos asistenciales adecuados. Sin embargo, el debate se enmarca fundamentalmente en aspectos clínicos, en la reorientación de los recursos disponibles y en las propias capacidades de los pacientes, teniendo en cuenta una óptica de "ética de mínimos" de no maleficencia y justicia (34), asumida profesionalmente más allá del mantenimiento del orden, del modelo biomédico imperante y de la discriminación de las personas.

En diciembre de 2012, a través del proyecto de Evaluación de la Estrategia de Salud Mental del Sistema Nacional de Salud (35), se volvió a plantear la no conveniencia de modificación de la LEC en relación a los TAI, existiendo un amplio consenso de los Comités Científico y e Institucional. Desde la coordinación del Comité Científico de la Estrategia, fueron aportados entonces una serie de argumentos clínicos en contra de los TAI (36), al no haberse demostrado su eficacia en la reducción de actos violentos e ingresos involuntarios en pacientes que padecen un TMG y que previamente hubiesen rechazado el tratamiento. Estas situaciones pueden abordarse a través de programas de detección precoz, tratamiento asertivo comunitario ó programas de tratamiento para pacientes con trastorno dual, además de reorientar y desarrollar plenamente los recursos disponibles de atención comunitaria.

Los tratamientos antipsicóticos, siendo relativamente eficaces, tienen efectos secundarios destacables que pueden condicionar la calidad de vida del paciente y conducir a los profesionales a serias encrucijadas éticas. La mayor parte de las Guías de Práctica Clínica utilizadas en la actualidad plantean explícitamente la necesidad de instaurar en los TMG Planes Individualizados de Tratamiento (PIT), que cuenten con la colaboración del paciente y contemplen, además de los fármacos, el entrenamiento en la adquisición de habilidades sociales, la psicoterapia, la rehabilitación cognitiva y distintas técnicas de intervención familiar (37).

\subsection{El Consentimiento Informado y la Planificación Anticipada de Decisiones}

El modelo de decisiones compartidas se está extendiendo en la práctica sanitaria y constituye un factor fundamental para una adecuada relación terapéutica. 
ORIGINALES Y REVISIONES

El modelo médico tradicional prioriza la opinión y las decisiones de quien posee el saber médico para determinar las intervenciones que deben realizarse, primando en muchas ocasiones el tratamiento psicofarmacológico, de modo que pueden estarse limitando los espacios de autonomía y decisión. Por esta razón, la creación de nuevos recursos y su diversificación, deben estar acompañados de prácticas destinadas al empoderamiento de las personas usuarias (19).

Son muchos los conflictos éticos que pueden suponer la vulneración de derechos en la atención a personas con enfermedad mental en intervenciones que no cuentan con el consentimiento del paciente o se realizan en contra de su voluntad; ¿se pueden hacer tratamientos involuntarios exclusivamente por indicación clínica, anulando cualquier tipo de elección del sujeto? (38) (hospitalización involuntaria, determinados tratamientos farmacológicos, medidas coercitivas como la contención mecánica ó el aislamiento...).

Un derecho habitualmente vulnerado tiene que ver con el consentimiento del tratamiento. Aunque la Ley de Autonomía del Paciente en España (39) vela por el derecho de disponer de información escrita o verbal de cualquier tipo de tratamiento en lenguaje y formato entendible, para posteriormente ser autorizado ó rechazado, pueden existir vulneraciones o interpretaciones que transgredan el principio de autonomía.

El derecho al consentimiento del tratamiento conlleva la evaluación de la competencia para decidir, la cual podría comprometer el libre ejercicio de la libertad (40). Históricamente se ha identificado enfermedad mental con capacidad limitada, pero enfermedad mental e incapacidad no deben ser planteadas como sinónimos. Toda persona es capaz mientras no se demuestre lo contrario y, además, no se puede decir de ninguna persona que sea plenamente incapaz o plenamente capaz para todos los actos de su vida (41). De esta manera, la capacidad no se entiende como un absoluto, así como su evaluación debe referirse a situaciones concretas en el ámbito clínico.

Una necesaria adaptación al nuevo paradigma que propugna la CDPD supone modificar la visión tradicional y paternalista de discapacidad de modo que nos basemos, no sólo en la persona, sino también en el entorno que dificulta el desarrollo de posibilidades reales. Si la situación transitoria de su enfermedad mental no lo permite, deberían proveerse instrumentos de apoyo que anticipen su voluntad cuando la persona se halle en disposición de hacerlo, de forma que siempre quede protegido su derecho a la autonomía (42).

En los últimos años, se está avanzando en la defensa de los derechos de autonomía y decisión de los pacientes, mediante la elaboración de documentos que puedan recoger las instrucciones previas o voluntades anticipadas para los momentos en los cuales no tengan control sobre sus vidas. Pese a que no existe una experiencia consolidada en todo el territorio español, están surgiendo experiencias 
muy interesantes, como las que se proponen desde la Federación Andaluza de Asociaciones de Usuarios/as de Salud Mental "En Primera Persona" (16).

Actualmente, en Andalucía están colaborando la Estrategia de Bioética del SSPA y el PISMA en líneas de trabajo que promuevan el respeto a los derechos de las personas que padecen un trastorno mental en relación a sus preferencias de tratamiento en situaciones de crisis con pérdida de competencia. En este sentido, se está valorando en la historia clínica digital, además del registro de la planificación anticipada de decisiones en el Plan Individualizado de Tratamiento (PIT), una nueva adaptación para la próxima edición de la Guía para hacer la Voluntad Vital Anticipada (43).

Una vez que el nivel de competencia de las personas que padecen un TMG fluctúa con el tiempo, puede plantearse entre el profesional y el paciente la formalización de una declaración previa personal y por escrito en la que se expresan, en el caso de sufrir una crisis, las preferencias negociadas de tratamiento sobre hospitalización, medicación, medidas coercitivas o cualquier otra intervención, contando preferiblemente con una tercera persona designada por el paciente para que actúe como mediadora.

La planificación anticipada de decisiones en Salud Mental, tanto en el ámbito hospitalario como ambulatorio, debe constituir una herramienta para proteger los derechos de las personas, mejorar la relación clínica y adaptar la asistencia a sus preferencias y necesidades (44). No debe nunca limitarse a la cumplimentación de un documento, sino formar parte de un proceso continuado de información, comunicación y apoyo a la toma de decisiones relacionadas con la atención de salud mental, e incluye el derecho de la persona a modificar o anular su contenido. Se requieren nuevos recursos, pero también mayores referencias empíricas y un cambio de cultura en profesionales, pacientes y familiares, siendo una alternativa al TAI más eficaz clínicamente y más coherente con la normativa actual sobre protección de los derechos y libertades fundamentales de las personas que padecen un TMG.

\section{Conclusiones}

Únicamente desde la óptica del respeto a los derechos humanos de las personas que padecen un trastorno mental, es posible luchar contra el estigma que padecen y puede intervenirse profesionalmente desde un enfoque asistencial y de cuidados centrado en la recuperación.

Este debate no se centra exclusivamente en relación a las personas que padecen un TMG. En los mal llamados Trastornos Mentales Menores, pueden también vulnerase derechos, de modo que se mantengan situaciones estigmatizantes, se 
ORIGINALES Y REVISIONES

obstaculice el proceso de recuperación y se agrave el sufrimiento personal, familiar y social.

Un procedimiento de planificación anticipada de decisiones en salud mental, integrado documentalmente a nivel clínico y adecuadamente garantista, constituye una herramienta decisiva para un proyecto terapéutico enfocado en la recuperación de las personas que padecen un TMG.

El diseño de un Plan Integral de Salud Mental que contenga como ejes transversales estos tres principios, derechos de las personas - lucha contra el estigma - enfoque de recuperación, podrá considerarse en todos sus términos un Plan Integral, Integrado, Intersectorial y con auténtica vocación de Salud Pública.

\section{Referencias Bibliográficas}

(1) The ITHACA Project Group (2010). ITHACA Toolkit for Monitoring Human Rights and General Health Care in Mental Health and Social Care Institutions. Health Service and Population Research Department, Institute of Psychiatry, King's College London, London. Disponible en: http://www.mdac.info/sites/mdac.info/files/ithaca_toolkit_english.pdf

(2) Consejería de Salud de la Junta de Andalucía. II Plan Integral de Salud Mental de Andalucía 2008-2012. Sevilla. La Consejería. 2008. Disponible en: http://www.sas.junta-andalucia. es/library/plantillas/externa.asp?pag=.././publicaciones/datos/473/pdf/PISMA_2008_2012.pdf

(3) Consejería de Salud de la Junta de Andalucía. Estrategia de Bioética del Sistema Sanitario Público Andaluz 2011-2014. Sevilla. La Consejería. 2011. Disponible en: http://www.juntadeandalucia.es/export/drupaljda/Libro_estrategia_Bioetica_SSPA.pdf

(4) ONU. Convención sobre los Derechos de las Personas con Discapacidad (CDPD). Nueva York. 2006.

(5) Consejería de Salud y Bienestar Social de la Junta de Andalucía. Derechos Humanos y Salud Mental en Andalucía. Sevilla. La Consejería. 2013. Disponible en: http:// www.sas.junta-andalucia.es/library/plantillas/externa.asp?pag=../../publicaciones/datos/551/pdf/ DerechosHumanosSaludMental20121203.pdf

(6) Consejería para la Igualdad y Bienestar Social de la Junta de Andalucía. II Plan de Acción Integral para Personas con Discapacidad de Andalucía 2011-2013. Sevilla. La Consejería. 2011. Disponible en: http://juntadeandalucia.es/export/drupaljda/Personas_Discapacidad_plan_ accion_integral_IIPAIPDA.pdf

(7) FAISEM, Fundación Pública Andaluza para la Integración Social del Enfermo Mental (2006). Plan de Calidad 2006 - 2010. http://www.faisem.es/ficheros/PlanCalidad.pdf

(8) Leal, J. (2009). Violencia, maltrato y sufrimiento en las instituciones. Asociación Española de Neuropsiquiatría. Violencia y salud mental. Ponencia del XXIV Congreso de la Asociación Española de Neuropsiquiatría. Cádiz, 3-6 de junio de 2009.

(9) Asociación de usuarios de Salud Mental de Cataluña (ADEMM). (2009). La relación entre las personas usuarias y profesionales en el ámbito de la salud mental. La opinión desde el 
colectivo de usuarios y profesionales. Proyecto ADEMM: Concienciación y empoderamiento de los usuarios. Divulgación de sus opiniones. Barcelona

(10) Libro Verde: Mejorar la Salud Mental de la población. Hacia una estrategia de la Unión Europea en Salud Mental. Comisión de las Comunidades Europeas, 2005.

(11) Díaz del Peral, D. El Estigma de la Enfermedad Mental. Tribuna Sanitaria. EL PAÍS. Publicado 15 de mayo de 2007.

(12) Escuela Andaluza de Salud Pública. Informe Evaluación de la intervención Lo hablamos. Sensibilización en las aulas. Granada. EASP. 2012. Disponible en: http://www.1decada4. es/adolescenciayestigma/lohablamos/Informe_Eval.pdf

(13) Consejería para la Igualdad y Bienestar Social de la Junta de Andalucía. Las personas con Trastornos Mentales Graves y los medios de comunicación. Sevilla. La Consejería. 2008.

(14) Bono del Trigo, A. Estigma y atención a la salud general de las personas con enfermedad mental. Estrategia contra el estigma 1decada4. Sevilla. Consejería de Salud. Junta de Andalucía. 2011

(15) FEAFES Andalucía http://www.feafesandalucia.org/images/stories/reivindicaciones_ FEAFES_A_2012.pdf

(16) Federación Andaluza de Asociaciones de usuarios/as Salud Mental. En Primera Persona. www.enprimerapersona.org

(17) WHO. QualityRights tool kit to assess and improve quality and human rights in mental health and social care facilities. Geneva, World Health Organization, 2012.

(18) Shepherd G., Boardman J. Making Recovery a Reality. London: Centre for Mental Health. 2008. Disponible en: http://www.centreformentalhealth.org.uk/pdfs/Making_recovery_a_ reality_policy_paper.pdf

(19) OMS. Empoderamiento del usuario de salud mental. Declaración de la Oficina Regional para Europa de la OMS. 2010.

(20) Romañach J. y Arnau MS. Omisiones bioéticas sobre discapacidad. Cuenta y Razón del Pensamiento Actual. 2004. 134: 65-72. Disponible en: http://www.cuentayrazon.org/revista/doc/134/ Num134_010.doc

(21) ONU. Declaración sobre los Derechos de las personas Impedidas. Nueva York: Asamblea General de las Naciones Unidas. 1975.

(22) ONU. Programa de Acción Mundial para las Personas Impedidas (Resolución 37/52). Nueva York: Asamblea General de las Naciones Unidas. 1982.

(23) ONU.Estándares sobre la igualdad de oportunidades para las Personas con Discapacidad (Resolución 48/96). Nueva York: Asamblea General de las Naciones Unidas. 1993.

(24) ONU. Principios para la Protección de las Personas con Retraso Mental, La enfermedad y la Mejora de la Atención de Salud Mental (Resolución 46/119). Nueva York: Asamblea General de las Naciones Unidas. 1991.

(25) OMS. Directrices sobre los derechos humanos de las personas con trastornos mentales. Ginebra: OMS. 1996.

(26) OMS. Atención a la salud mental: diez principios básicos. Ginebra: OMS. 1996.

(27) Recomendación Rec 10 del Consejo de Europa, relativa a la protección de los derechos humanos y a la dignidad de las personas aquejadas de trastornos mentales, de 22.9.2004.

(28) United Nations Human Rights FORGOTTEN EUROPEANS-FORGOTTEN RIGHTS. Oficina del Alto Comisionado de Derechos Humanos para Europa. 2010. 
ORIGINALES Y REVISIONES

(29) Instrumento de Ratificación de la Convención sobre los derechos de las personas con discapacidad. Boletín Oficial del Estado, 21 de abril de 2008, núm. 96, p.20648-20658.

(30) OMS. Manual de Recursos de la OMS sobre Salud Mental, Derecho Humanos y Legislación. Ginebra: OMS. 2006.

(31) Medrano Albéniz J. Tratamiento ambulatorio involuntario. News. (Soc. Esp. Psiquiatr. Legal) 2005; 12. Disponible en: http://www.psiquiatrialegal.org/TAI2.htm

(32) AEN http://www.portalsaludmental.com/Article326.html Noviembre 2006.

(33) Romero J., Gorrotxategui M. Aproximación al debate sobre el tratamiento ambulatorio involuntario desde una perspectiva bioética. Rev. Asoc. Esp. Neuropsiq. 2009; 104:423-436.

(34) Gracia D. La deliberación moral: el método de la ética clínica. Med Clin (Barc) 2001; 117: 18-23. Disponible en: http://dx.doi.org/10.1016/S0025-7753(01)71998-7

(35) Ministerio de Sanidad y Consumo. Estrategia en Salud Mental del Sistema Nacional de Salud 2009-2013. Madrid: Ministerio de Sanidad y Consumo; 2007. Disponible en:

http://www.msssi.gob.es/organizacion/sns/planCalidadSNS/docs/saludmental/ SaludMental2009-2013.pdf

(36) Gómez Beneyto M. Informe sobre el Tratamiento Ambulatorio Involuntario. Estrategia en Salud Mental del Sistema Nacional de Salud. Madrid 2012.

(37) Consejería de Salud de la Junta de Andalucía. Proceso Asistencial Integrado Trastorno Mental Grave. Sevilla. La Consejería. 2006

(38) Seoane, JA. Derecho y Salud Mental. Capacidades, derechos, justicia. Cuad. Psiquiatr. Comunitaria. 2006; 6: 21-38.

(39) Boletín Oficial del Estado. Ley 41/2002, de 14 de noviembre, básica reguladora de la autonomía del paciente y de derechos y obligaciones en materia de información y documentación clínica. BOE 274, de 15 de noviembre de 2002,p.p. 40136-40132

(40) Cartagena FS. y Manrique MH. Los comités de ética asistencial en el campo de la salud mental. Norte de Salud Mental. 2010.3(10).

(41) Simón-Lorda P.: "Diez mitos en torno al consentimiento informado" An. Sist. Sanit. Navar.2006, vol 29 Suplemento 3.

(42) Simón-Lorda P.: "La capacidad de los pacientes para tomar decisiones: una tarea todavía pendiente". Rev. Asoc. Esp. Neuropsiq., 2008 vol. XXVIII, n 102, pp. 325-348

(43) Consejería de Salud de la Junta de Andalucía. Guía para hacer la Voluntad Vital Anticipada. Sevilla. La Consejería. 2012. Disponible en: https://rv2.csalud.junta-andalucia.es/salud/ rv2/descargasCiudadano. action? $=0 \&$ idMenu $=58$

(44) Gorrotxategi M, Cuesta JR. Voluntades psiquiátricas: Una herramienta terapéutica. Norte de salud mental 2012; 10(42):11-17 